\title{
Myeloid-derived suppressor cells and their "inconvenient" plasticity
}

\author{
David Escors',2, Grazyna Kochan ${ }^{1 *}$
}

'Navarrabiomed-Fundación Miguel Servet. Complejo Hospitalario de Navarra. Irunlarrea 3, 31008, Pamplona. Navarra. Spain. ${ }^{2}$ Division of Infection and Immunity. University College London, 5 University Street, WC1E 6JF London, United Kingdom.

Article Info

\section{Article Notes}

Received: March 26, 2018

Accepted: April 19, 2018

\section{*Correspondence:}

Dr. Grazyna Kochan, Navarrabiomed-Fundación Miguel Servet. Complejo Hospitalario de Navarra. Irunlarrea 3 , 31008, Pamplona. Navarra. Spain,

E-mail: grazyna.kochan@navarra.es

(c) 2018 Kochan $\mathrm{G}$. This article is distributed under the terms of the Creative Commons Attribution 4.0 International License.

\section{ABSTRACT}

Myeloid-derived suppressor cells (MDSCs) comprise certain types of myeloid subsets with strong immunosuppressive activities, which expand at high levels in pathological conditions such as cancer. A major drawback in the study of MDSCs is the extraordinary plasticity of the myeloid lineage that hampers the identification of MDSC subsets, especially in humans. Here we provide a brief overview on MDSCs, their differentiation and the current difficulties in classifying these immunosuppressive subsets.

\section{Myeloid-derived suppressor cell subsets}

Myeloid cells comprise a highly diverse population involved in the maintenance of the equilibrium of our immune system. They undergo major phenotypic changes (activation) as a result of engaging with pathogen- or danger-associated stimuli (pathogen associated molecular pattern (PAMPs) and danger associated molecular pattern (DAMPs). Once the source of stimuli disappear activation terminates. However, this is not the case of inflammatory chronic diseases or cancer, disorders that usually maintain continuous inflammatory stimuli and sustained exposure to different antigens. In these conditions, myeloid reprogramming takes place, leading to the differentiation of myeloid cells with different characteristics as those found in non-pathological conditions. Both in murine cancer models and in humans with neoplastic disorders, tumors disrupt the whole systemic homeostasis by producing a wide collection of cytokines, growth factors and chemokines that are distributed through blood and lymph ${ }^{1,2}$. These cytokines reach the bone marrow and significantly perturb the physiological myelopoiesis, especially when the tumor burden is high. Amongst these soluble mediators, tumors usually produce high levels of GM-CSF, M-CSF, IL4, IL6, IL18, IL13, prostaglandins, IL10 and TGF- $\beta^{3-9}$. High levels of GM-CSF are critical for the differentiation of MDSCs instead of myeloid cells such as dendritic cells, monocytes, granulocytes or macrophages ${ }^{10,11}$.

The first reports on the accumulation of myeloid cells within tumors as a sign of poor prognosis were published in the early $1970 \mathrm{~s}^{12}$. Since then numerous studies have clearly demonstrated that systemic expansion and accumulation of myeloid populations with suppressive activity is associated with tumor progression and metastasis, both in preclinical and clinical cancer models. Then, in 2007 it was proposed that myeloid cells with suppressive activities should be covered by a common denomination; myeloid 
derived suppressor cells (MDSC). This was an attempt to acknowledge the existence of these cells as distinct entities from those found in non-pathological conditions.

Since the 1990s, numerous studies and extensive work in this subject uncovered two distinct MDSC subsets, well characterized in murine models: monocitic (M)MDSC present a distinct phenotype which reminds to that of inflammatory monocytes (CD11b+Ly6G-Ly6C $\left.C^{\text {hi }}\right)$ while granulocytic (G) or polymorphonuclear (PMN)MDSC show a phenotype closely related to granulocytes $\left(\mathrm{CD} 11 \mathrm{~b}+\mathrm{Ly} 6 \mathrm{G}+\mathrm{Ly} 6 \mathrm{C}^{\mathrm{l}}\right)$. It has to be remarked that these two phenotypes are nearly exactly the same as found in non-pathological counterparts, and the truly differential characteristic is the high suppressive activities of MDSCs towards other immune cell types such as activated T cells. ${ }^{1}$ The acquisition of strong immunosuppressive activities is the probable result of major transcriptomic and metabolic changes, which in turn are regulated by a distinct core of activated protein kinases including ERK, AKT and PKCs

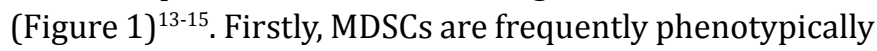
"immature", meaning that they express on the surface low levels of major histocompatibility molecules (MHC) and some (but not all) co-stimulatory molecules ${ }^{11,16}$. This absence of MHC molecules on their surface prevents efficacious antigen presentation to $\mathrm{T}$ cells. However, MDSCs do inhibit previously-activated $\mathrm{T}$ cells through a variety of mechanisms (Figure 2), which include depletion of essential aminoacids such as glutamine, production of immunosuppressive cytokines such as TGF- $\beta$ or IL-10 ${ }^{11,17}$,

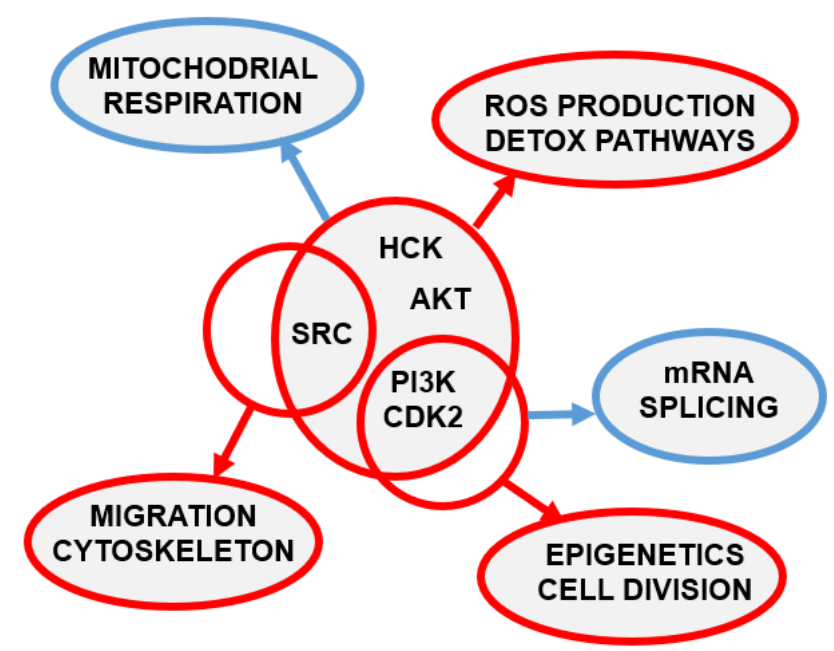

Figure 1. A core of kinase-regulated interactomes regulates diverse MDSC functions. The figure lists in the center kinases that are specifically up-regulated in MDSCs. The relationship between the kinases and the cellular functions regulated by them are indicated with Venn diagrams. In this way, specific group of kinases are associated together within circles communicated with cellular functions with arrows, as indicated. Within red circles, MDSC functions that are up-regulated in MDSCs. Within blue circles, MDSC functions that are down-modulated in MDSCs. expression of T cell inhibitory molecules such as PD-L $1^{13,18}$ and the production of nitric oxide (NO) by constitutive expression of iNOS. NO production causes the nitrosylation of a high number of intracellular proteins, a posttranslational modification that alters their activities ${ }^{11,19}$.

Nevertheless, the presence of specific surface markers made feasible their purification from murine models and their characterization by genomic ${ }^{20}$ and proteomic approaches ${ }^{13,14}$. In vitro differentiation systems and also some in vivo studies in murine cancer models allowed the demonstration that granulocytic MDSCs comprise the final stage of monocytic MDSC maturation ${ }^{2111}$.

Even though there is certainly a lack of specific markers exclusively owned only by MDSC populations, a variety of panels of markers characterising human MDSC monocytic and granulocytic populations have been published. Indeed, this has been probably the result of trying to characterize the phenotype of human MDSC counterparts. While the phenotype of G-MDSC and M-MDSC in mice is certainly straightforward (albeit of their similarities with nonpathological myeloid subsets), this is not the case in humans. Early on, human monocitic MDSC were defined as CD11 $\mathrm{b}^{+}$ $\mathrm{CD}_{14}{ }^{+} \mathrm{HLA}^{-D R^{-/ 10}} \mathrm{CD}^{-} 5^{-}$, in resemblance to the phenotype of human inflammatory monocytes, and similarly to murine G-MDSC. Likewise, human G-MDSC subsets were defined by a phenotype closely resembling that of human neutrophils $\left(\mathrm{CD} 11 \mathrm{~b}^{+} \mathrm{CD} 14^{-} \mathrm{CD} 15^{+} \mathrm{CD} 6 \mathrm{~b}^{+}\right)^{22,23}$. Again, in resemblance of the murine model with G-MDSCs resembling granulocytes. Nevertheless, the lookout for novel MDSC-specific markers has not ceased, and recently LOX1 was described as specific marker of G-MDSC ${ }^{24}$.

As commented above, the particularity of MDSC resides on their strong suppressive activities, which are absent in their non-pathological counterparts. Since the identification of their phenotypical markers, these cells have been isolated or produced in vitro, and numerous studies have been characterizing their functional activities. These studies have uncovered a wide range of suppressive mechanisms that MDSCs employ to suppress the effector activities and proliferation of different immune cell populations, most extensively in activated $\mathrm{T}$ cells. Many of these mechanisms are shared by previously-known immunosuppressive pathways exerted by, for example, M2 macrophages or even regulatory T cells (Tregs).

In recent years there is a growing body of literature unveiling the role of MDSCs in non-neoplastic pathological conditions such as autoimmune and many infectious diseases, graft versus host response or in maternal-foetal tolerance (reviewed elsewhere ${ }^{1,25}$ ).

\section{Myeloid plasticity as the main "inconvenience"}

The situation nowadays on MDSCs is certainly one of confusion ${ }^{2}$. By no means, many immunologists and 


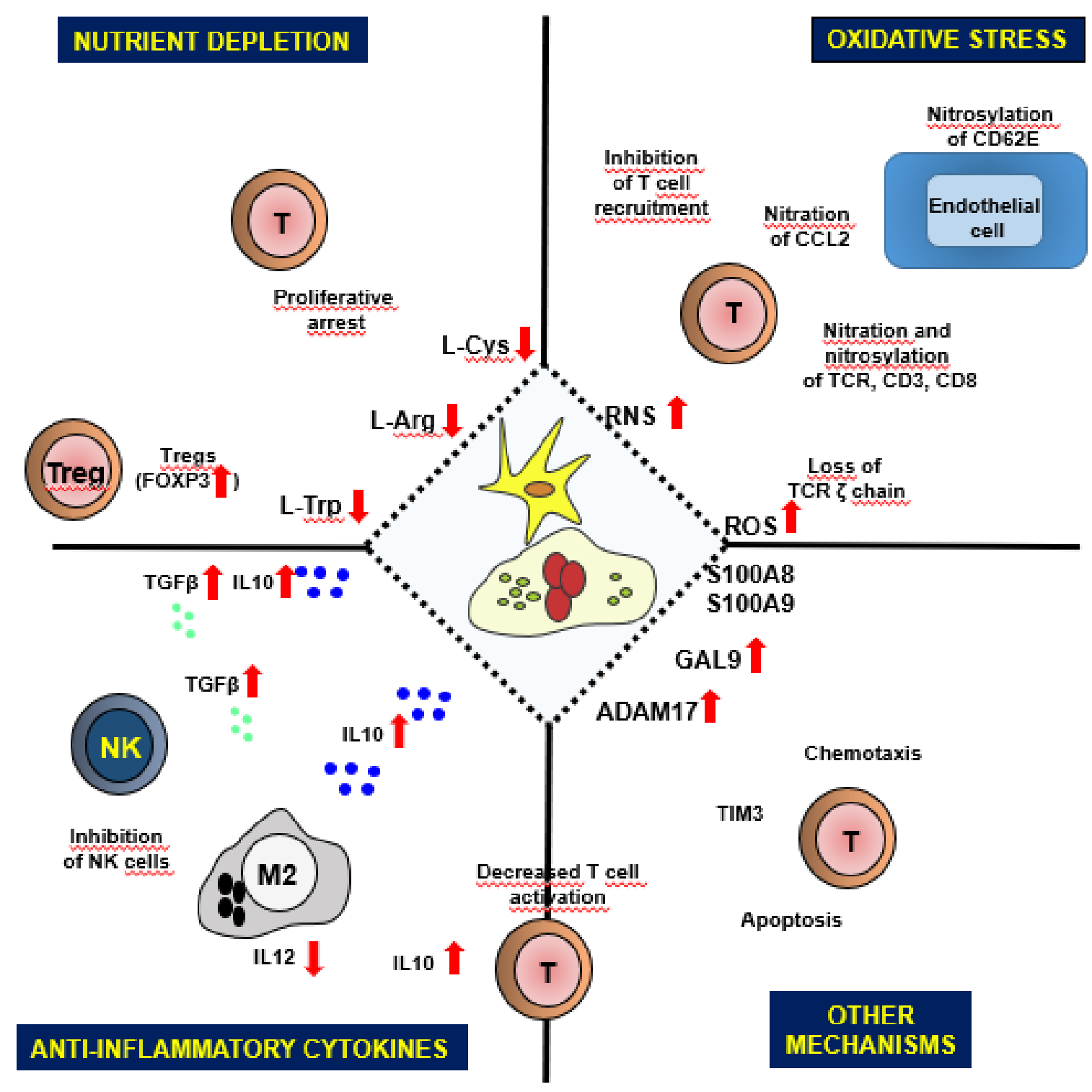

Figure 2. MDSC-dependent immunosuppressive mechanisms. Myeloid derived suppressor cells affect the antitumor action of the immune system using several mechanisms as schematically shown in the figure. Nutrient depletion: MDSCs deplete essential amino acids (Arg, Trp and Cys) leading to inhibition of T cell proliferation and enhancement of FoxP3 positive regulatory T cells. Oxidative Stress: Reactive nitrogen species (RNS) nitrate CCL2 that is required for T cell recruitment to the tumor site. CD62E nitrosylation on endothelial cells also blocks T cell recruitment to the tumor site. Nitration and nitrosylation of CD3, CD8 and T cell receptor (TCR) diminish the effector function of T cells. Generation of high levels of reactive oxygen species (ROS) causes the loss of the TCR abolishing T cell effector function. Antiinflammatory cytokines: MDSCs produce high levels of TGF- $\beta$ and IL10 which favor Treg differentiation and functions. TGF- $\beta$ affects the production of IFN- $\gamma$ by NK cells. High levels of IL10 induce the M2 macrophage phenotype and further production of IL10 by macrophages within the tumor. Furthermore, IL12 production is down-modulated. In addition, these changes in the cytokine milieu within tumors disturb T cell activation. Other suppressive mechanisms: MDSCs produce proteins such as S100A8 and A100A9, responsible for attracting MDSCs into tumor sites and increasing their suppressive phenotype. ADAM17 degrades CD62L levels on the surface of T cells, which is required for homing of T cells to proximal lymphatic nodes and for their activation. Galectin 9 on the surface of MDSCs interacts with TIM3 on the surface of $T$ cells causing their apoptosis.

researchers do not accept their existence as specific myeloid entities different from the classical myeloid cell types. And even those who acknowledge their existence do not agree in many things, including their phenotype, origins and relationships amongst these cell types ${ }^{22}$. Why is that?
Indeed, the culprit here is the extraordinary differentiation plasticity of the myeloid lineage both in murine models and humans. This is the main "inconvenience". This "inconvenient" situation also occurred some time ago with Tregs, which also lacked specific markers of their 
own during the $70 \mathrm{~s}$ and $80 \mathrm{~s}^{26}$. Only when Tregs were defined as CD25high Foxp3 positive, they could be studied, characterized, and more importantly, accepted by the scientific and medical community.

This is certainly not the case yet with MDSCs. The immense plasticity of myeloid populations from the bone marrow to peripheral tissues subjected under the enormous pressure of tumor-derived factors makes it difficult to clearly discriminate between MDSCs, neutrophils, monocytes or tolerogenic dendritic cells. As a consequence, some authors have proposed the classification of all myeloid populations with suppressive activity under the common denomination of myeloid regulatory cells ${ }^{27,28}$. But again, it is unclear whether this common denominator is yet another way to not addressing the main problem with MDSCs. Their enormous plasticity.

\section{Myeloid plasticity in pathological situations}

Confusion due to the remarkable plasticity of myeloid cells starts with the MDSCs themselves in the murine system. Since their identification and phenotypic characterization, monocytic and granulocytic MDSCs have been considered to be lineages apart rather than belonging to the same "cell type" under distinct differentiation stages $^{9,29}$. This distinction has been maintained through most of the published works, and remains to be so nowadays. This is likely caused by their significant phenotypic resemblance to monocytes and granulocytes in non-pathological conditions. Therefore, it was only logical to assume that monocytic MDSCs came from the same differentiation route than monocytes and $\mathrm{DCs}^{30}$, while granulocytic MDSCs came from the differentiation pathway of granulocytes ${ }^{31}$. This distinction is of relevance, as differentiation of monocytic and granulocytic lineages takes place early in myelopoiesis at the granulocytemonocyte progenitor (GMP) stage. Expression at this stage of PU.1 and IRF8 transcription factors leads GMP towards the monocytic lineage differentiation, which includes DCs, monocytes and macrophages ${ }^{32-34}$. In contrast, expression of $\mathrm{c} / \mathrm{EBP} \alpha$ expression causes GMP to differentiate to the granulocytic lineage $\mathrm{e}^{35,36}$. However, MDSC differentiation cannot be compared to physiological myelopoiesis, as it takes place under specific circumstances. Differentiation in neoplastic conditions resembles much more myelopoiesis under stress, which favors fast expansion of granulocytes through an alternative non-steady state differentiation route regulated by $\mathrm{c} / \mathrm{EBP} \beta^{37,38}$. Indeed, at least in murine models it seems that granulocytic MDSCs are the terminal differentiation stage of monocytic MDSC subsets. This was demonstrated in vivo in tumor-bearing mice, in which G-MDSCs quickly differentiated from M-MDSCs by epigenetic silencing of retiblastoma expression, possibly regulated by hypoxia ${ }^{39}$. The demonstration that this was indeed the case came from in vitro differentiation of
MDSCs from murine bone marrow. Purified monocytic MDSCs quickly converted to granulocytic MDSCs by strong up-regulation of Ly6 $6 \mathrm{G}^{11}$, which in turn could be regulated by the interplay of distinct signalling pathways regulating different aspects of MDSC functions ${ }^{13}$. A key question remains whether the same differentiation pathways are present in the human system. At least in the murine system, it has been proposed that MDSCs arise probably from a CD11b+ Ly6Cneg Ly6Gneg GMP-like or MDP-like precursor that will differentiate into M-MDSC. Then, following the activity of the $\mathrm{c} / \mathrm{EBP} \beta$ transcription factor, M-MDSC will quickly differentiate into G-MDSC as early as in the bone marrow or within the tumor environment through the activity of IRF8 ${ }^{40-43}$.

A strong drawback of studying MDSC differentiation in humans is the lack of efficacious in vitro differentiation systems. In most cases, "MDSCs" or rather "myeloid populations" are monitored in peripheral blood, or MDSCs differentiated from adherent monocytes obtained from peripheral blood. Therefore, it is rather hard to identify the signalling pathways regulating human MDSC differentiation when most of the times early precursors are not utilized ${ }^{16,44}$. It goes without saying that myeloid populations infiltrating the tumor are rather heterogeneous, and sometimes it is quite challenging to identify myeloid cells as MDSCs, macrophages or tolerogenic dendritic cells ${ }^{16,45}$.

MDSC plasticity is also evident by their intrinsic capacities of becoming immunostimulatory. Thus, intratumor MDSCs can quickly convert to immunostimulatory myeloid cells with strong $\mathrm{T}$ cell stimulatory activities, and turn an immunosuppressive tumor environment into an efficacious anti-tumor response. This is perfectly exemplified by the expression of IL-12, a cytokine with potent T cell stimulatory activities. Hence, modification of murine MDSCs with lentivectors expressing IL-12 can turn these cells into potent antigen-presenting cells and raise effective anti-tumor immune responses ${ }^{46,47}$. This effect is potentiated by combining IL-12 expression with a PD-L1-silencing microRNA that enhances antigen presentation by non-professional antigen presenting cells ${ }^{47}$, and inhibits tumor growth in vivo by delaying cancer cell proliferation and sensitizing these cells to cytotoxicity by $\mathrm{T}$ cells ${ }^{48}$. IL-12 expression in several cancer models also leads to an increase of CD80, MHC class II and other maturation molecules in MDSCs, as well as an increase in myeloid DCs ${ }^{49,50}$.

\section{Conclusions}

It seems evident that MDSC do really exist. There is overwhelming evidence on their differentiation and their clear detrimental effects in pathologies such as cancer. However, whether MDSCs can be considered a lineage of their own is still rather hard. This is caused by the 
enormous plasticity of the myeloid lineage, and the high inter-conversion capabilities of one myeloid cell type towards another depending on their environment and interactions with other cell types. Even their characteristic immunosuppressive properties can also be found (or induced) in non-MDSC counterparts. Nevertheless, it cannot be argued that MDSCs are real entities in murine models. However, human MDSCs, or rather their existence, is rather complicated to demonstrate. There is a lack of in vitro well-defined differentiation system that may allow their analysis by genomic and proteomic techniques. Indeed, most of the evidence shows an extraordinary variety of myeloid cell types in humans ${ }^{51}$, which hampers rather than sheds light on human MDSC differentiation and their role in cancer. Nevertheless, it is likely that MDSCs do exist in humans, although their phenotypic differentiation, origin and functions may differ significantly from the murine counterparts.

\section{Conflict of interest statement}

The authors declare no conflicts of interest

\section{Acknowledgements}

Our group is supported by project funds granted by Instituto de Salud Carlos III, Spain (FIS project grant PI17/02119), Asociación Española Contra el Cáncer (AECC, PROYE16001ESCO) and a "Precipita" Crowdfunding grant (Fundación Española para la Ciencia y Tecnología, FECYT), and "Obra Social" La Caixa (Spain).

\section{References}

1. Veglia F, Perego M, Gabrilovich D. Myeloid-derived suppressor cells coming of age. Nat Immunol. 2018; 19: 108-119, doi:10.1038/ s41590-017-0022-x.

2. Tcyganov E, Mastio J, Chen E, et al. Plasticity of myeloid-derived suppressor cells in cancer. Curr Opin Immunol. 2018; 51: 76-82, doi:10.1016/j.coi.2018.03.009.

3. Highfill SL. Bone marrow myeloid-derived suppressor cells (MDSCs) inhibit graft-versus-host disease (GVHD) via an arginase1-dependent mechanism that is up-regulated by interleukin-13. Blood. 2010; 116: 5738-5747, doi:10.1182/blood-2010-06-287839 blood-2010-06-287839 [pii].

4. Veltman JD. COX-2 inhibition improves immunotherapy and is associated with decreased numbers of myeloid-derived suppressor cells in mesothelioma. Celecoxib influences MDSC function. BMC Cancer. 2010; 10: 464, doi:10.1186/1471-2407-10-464.

5. Lim HX, Hong HJ, Cho D, et al. IL-18 enhances immunosuppressive responses by promoting differentiation into monocytic myeloidderived suppressor cells. J Immunol. 2014; 193: 5453-5460.

6. Chen MF. IL-6-stimulated CD11b+ CD14+ HLA-DR- myeloid-derived suppressor cells, are associated with progression and poor prognosis in squamous cell carcinoma of the esophagus. Oncotarget. 2014; 5: 8716-8728.

7. Obermajer N, Muthuswamy R, Lesnock J, et al. Positive feedback between PGE2 and COX2 redirects the differentiation of human dendritic cells toward stable myeloid-derived suppressor cells. Blood. 2013; 118: 5498-5505.

8. Mandruzzato S. IL4Ralpha+ myeloid-derived suppressor cell expansion in cancer patients. J Immunol. 2009; 182: 6562-6568, doi:10.4049/jimmunol.0803831.

9. Youn JI, Nagaraj S, Collazo M, et al. Subsets of myeloid-derived suppressor cells in tumor-bearing mice. J Immunol. 2008; 181: 57915802.

10. Morales JK, Kmieciak M, Knutson KL, et al. GM-CSF is one of the main breast tumor-derived soluble factors involved in the differentiation of CD11b-Gr1- bone marrow progenitor cells into myeloid-derived suppressor cells. Breast Cancer Res Treat. 2010; 123: 39-49.

11. Liechtenstein T. A highly efficient tumor-infiltrating MDSC differentiation system for discovery of anti-neoplastic targets, which circumvents the need for tumor establishment in mice. Oncotarget. 2014; 5: 7843-7857, doi:10.18632/oncotarget.2279.

12. Talmadge JE, Gabrilovich DI. History of myeloid-derived suppressor cells. Nature reviews. 2013; 13: 739-752.

13. Gato-Canas M. A core of kinase-regulated interactomes defines the neoplastic MDSC lineage. Oncotarget. 2015; 6: 27160-27175, doi:10.18632/oncotarget.4746.

14. Gato M. Drafting the proteome landscape of myeloid-derived suppressor cells. Proteomics. 2016; 16: 367-378, doi:10.1002/ pmic.201500229.

15. Barbosa CM, Bincoletto C, Barros CC, et al. PLCgamma2 and PKC are important to myeloid lineage commitment triggered by M-SCF and G-CSF. J Cell Biochem. 2014; 115: 42-51.

16. Ibanez-Vea M. Myeloid-Derived Suppressor Cells in the Tumor Microenvironment: Current Knowledge and Future Perspectives. Arch Immunol Ther Exp (Warsz). 2017. doi:10.1007/s00005-017-0492-4.

17. Dufait I. Ex vivo generation of myeloid-derived suppressor cells that model the tumor immunosuppressive environment in colorectal cancer Oncotarget. 2015; 6: 12369-12382.

18. Karwacz K. PD-L1 co-stimulation contributes to ligand-induced T cell receptor down-modulation on CD8(+) T cells. EMBO Mol Med. 2011; 3: 581-592, doi:10.1002/emmm.201100165.

19. Ibanez-Vea M, Huang H, Martínez de Morentin X, et al. Characterization of Macrophage Endogenous S-Nitrosoproteome Using a CysteineSpecific Phosphonate Adaptable Tag in Combination with TiO2 Chromatography. Journal of proteome research. 2018; doi:10.1021/ acs.jproteome.7b00812.

20. Fridlender ZG, Sun J, Mishalian I, et al. Transcriptomic analysis comparing tumor-associated neutrophils with granulocytic myloidderived suppressor cells and normal neutrophils. PLoS ONE. 2012; 7: e31524.

21. Corzo CA, Condamine T, Lu L, et al. HIF-1alpha regulates function and differentiation of myeloid-derived suppressor cells in the tumor microenvironment. J Exp Med. 2010; 207: 2439-2453, doi:10.1084/ jem.20100587 jem.20100587 [pii].

22. Bronte V, Brandau S, Chen SH, et al. Recommendations for myeloidderived suppressor cell nomenclature and characterization standards. Nature communications. 2016; 7: 12150, doi:10.1038/ ncomms12150.

23. Mandruzzato S, Brandau S, Britten CM, et al. Toward harmonized phenotyping of human myeloid-derived suppressor cells by flow cytometry: results from an interim study. Cancer Immunol Immunother. 2016; 65: 161-169, doi:10.1007/s00262-015-1782-5.

24. Condamine T, Dominguez GA, Youn JI, et al. Lectin-type oxidized LDL receptor-1 distinguishes population of human polymorphonuclear myeloid-derived suppressor cells in cancer patients. Science immunology. 2016; 1: doi:10.1126/sciimmunol.aaf8943.

25. Kwak Y, Kim HE, Park SG. Insights into Myeloid-Derived Suppressor Cells in Inflammatory Diseases. Arch Immunol Ther Exp (Warsz). 2015; 63: 269-285, doi:10.1007/s00005-015-0342-1. 
26. Escors D. Tumour immunogenicity, antigen presentation and immunological barriers in cancer immunotherapy. New journal of science. 2014 ; 2014. doi:10.1155/2014/734515.

27. Gutkin DW, Shurin MR. Clinical evaluation of systemic and local immune responses in cancer: time for integration. Cancer Immunol Immunother. 2014; 63: 45-57. doi:10.1007/s00262-013-1480-0.

28. Zhong H, Gutkin DW, Han B, et al. Origin and pharmacological modulation of tumor-associated regulatory dendritic cells. Int J Cancer. 2014; 134: 2633-2645, doi:10.1002/ijc.28590.

29. Tsiganov EN, Verbina EM, Radaeva TV, et al. Gr-1dimCD $11 b+$ Immature Myeloid-Derived Suppressor Cells but Not Neutrophils Are Markers of Lethal Tuberculosis Infection in Mice. J Immunol. 2014; 192: 4718-4727. doi:10.4049/jimmunol.1301365.

30. Lutz MB, Kukutsch NA, Menges M, et al. Culture of bone marrow cells in GM-CSF plus high doses of lipopolysaccharide generates exclusively immature dendritic cells which induce alloantigen-specific CD4 T cell anergy in vitro. Eur J Immunol. 2000; 30: 1048-1052.

31. Pillay J, Kamp VM, van Hoffen E, et al. A subset of neutrophils in human systemic inflammation inhibits T cell responses through Mac-1. J Clin Invest. 2012; 122: 327-336, doi:10.1172/JCI57990 57990 [pii].

32. Yang ZF, Drumea K, Cormier J, et al. GABP transcription factor is required for myeloid differentiation, in part, throught its control of Gfi-1 expression. Blood. 2011; 118: 2243-2253.

33. Friedman AD. C/EBPalpha in normal and malignant myelopoiesis. Trans Int J Hematol. 2015; 101: 330-341.

34. Tamura T, Kurotaki D, Koizumi S. Regulation of myelopoiesis by the transcription factor IRF8. Int J Hematol. 2015; 101: 342-351.

35. Geest CR, Buitenhuis M, Laarhoven AG, et al. p38 MAP kinase inhibits neutrophil development through phosphorylation of C/EBPalpha on serine 21. Stem Cells. 2009; 27: 2271-2282.

36. Hong S, Skaist AM, Wheelan SJ, et al. AP-1 protein induction during monopoiesis favors C/EBP:AP-1 heterodimers over C/EBP homodimerization and stimulates FosB transcription. J Leukoc Biol. 2011; 90: 643-651.

37. Hirai H, Yokota A, Tamura A, et al. Non-steady-state hematopoiesis is regulated by the C/EBPbeta transcription factor. Cancer Sci. 2015. doi: 10.1111/cas.12690.

38. Marigo I, Bosio E, Solito S, et al. Tumor-induced tolerance and immune suppression depend on the C/EBPbeta transcription factor. Immunity 2010; 32: 790-802, doi:10.1016/j.immuni.2010.05.010 S10747613(10)00202-5 [pii].

39. Youn JI, KumarV, Collazo M, etal.Epigenetic silencing of retinoblastoma gene regulates pathologic differentiation of myeloid cells in cancer. Nat Immunol. 2013; 14: 211-220, doi:10.1038/ni.2526 ni.2526 [pii].
40. Netherby CS, Messmer MN, Burkard-Mandel L, et al. The Granulocyte Progenitor Stage Is a Key Target of IRF8-Mediated Regulation of Myeloid-Derived Suppressor Cell Production. J Immunol. 2017; 198: 4129-4139, doi:10.4049/jimmunol.1601722.

41. Netherby CS, Abrams SI. Mechanisms overseeing myeloid-derived suppressor cell production in neoplastic disease. Cancer Immunol Immunother. 2017; 66: 989-996, doi:10.1007/s00262-017-1963-5.

42. Abrams SI, Netherby CS, Twum DY, et al. Relevance of Interferon Regulatory Factor-8 Expression in Myeloid-Tumor Interactions. Journal of interferon \& cytokine research : the official journal of the International Society for Interferon and Cytokine Research. 2016; 36: 442-453, doi:10.1089/jir.2015.0174.

43. Jiang M, Chen J, Zhang W, et al. Interleukin-6 Trans-Signaling Pathway Promotes Immunosuppressive Myeloid-Derived Suppressor Cells via Suppression of Suppressor of Cytokine Signaling 3 in Breast Cancer. Front Immunol. 2017; 8: 1840, doi:10.3389/fimmu.2017.01840.

44. Sinha P, Clements VK, Fulton AM, et al. Prostaglandin E2 promotes tumor progression by inducing myeloid-derived suppressor cells. Cancer Res. 2007; 67: 4507-4513, doi:10.1158/0008-5472.CAN-06-4174.

45. Elliott LA, Doherty GA, Sheahan K, et al. Human Tumor-Infiltrating Myeloid Cells: Phenotypic and Functional Diversity. Front Immunol. 2017; 8: 86, doi:10.3389/fimmu.2017.00086.

46. Liechtenstein T, Perez-Janices N, Bricogne C, et al. Immune modulation by genetic modification of dendritic cells with lentiviral vectors. Virus Res. 2013; 176: 1-15, doi:10.1016/j.virusres.2013.05.007.

47. Liechtenstein T, Perez-Janices N, Blanco-Luquin I, et al. Antimelanoma vaccines engineered to simultaneously modulate cytokine priming and silence PD-L1 characterized using ex vivo myeloidderived suppressor cells as a readout of therapeutic efficacy. Oncoimmunology. 2014; 3: e29178.

48. Gato-Canas M, Zuazo M, Arasanz H, et al. PDL1 Signals through Conserved Sequence Motifs to Overcome Interferon-Mediated Cytotoxicity. Cell Rep. 2017; 20: 1818-1829, doi:10.1016/j. celrep.2017.07.075.

49. Thaci B, Ahmed AU, Ulasov IV, et al. Depletion of myeloid-derived suppressor cells during interleukin-12 immunogene therapy does not confer a survival advantage in experimental malignant glioma. Cancer Gene Ther. 2014; 21: 38-44, doi:10.1038/cgt.2013.81 cgt201381 [pii].

50. Steding CE, Wu ST, Zhang Y, et al. The role of interleukin-12 on modulating myeloid-derived suppressor cells, increasing overall survival and reducing metastasis. Immunology. 2011; 133: 221-238, doi:10.1111/j.1365-2567.2011.03429.x.

51. Villani AC, Satija R, Reynolds G, et al. Single-cell RNA-seq reveals new types of human blood dendritic cells, monocytes, and progenitors. Science. 2017; 356: doi:10.1126/science.aah4573. 